\title{
Microbial Integration on Player Experience of Hybrid Bio-Digital Games
}

\author{
Raphael Kim ${ }^{1(凶)}$, Siobhan Thomas ${ }^{2}$, Roland van Dierendonck ${ }^{3}$ \\ Antonios Kaniadakis ${ }^{1}$, and Stefan Poslad ${ }^{1}$ \\ ${ }^{1}$ Queen Mary University, London, United Kingdom \\ ${ }^{2}$ London South Bank University, London, United Kingdom \\ ${ }^{3}$ Studio Roland van Dierendonck, Amsterdam, The Netherlands \\ $\bowtie$ r.s.kim@qmul.ac.uk
}

\begin{abstract}
Hybrid bio-digital games physically integrate non-human, living organisms into computer gaming hardware and software. Whilst such type of game can add novelty value, the positive impact of the added biological element on player experience has not yet been verified quantitatively. We conducted a study involving two groups of 20 participants, to compare player experiences of two versions of a video game called Mould Rush, which relies on the growth patterns of micro-organisms commonly known as 'mould'. Results from self-reporting Game Experience Questionnaire (GEQ) showed that the group who played the version of Mould Rush that integrated real mould, had produced significantly higher mean GEQ scores $(p<.001)$ on the following dimensions: Positive Affect; Sensory and Imaginative Immersion; Positive Experience; and Returning to Reality. Furthermore, results from participant interviews indicated that the slowness of mould growth was enjoyed by those who played real-mould-integrated version of Mould Rush. Contrastingly, the slowness was perceived as a negative feature for those who played the game without integrated mould. We discuss the implications and limitations of all of our findings.
\end{abstract}

Keywords: Hybrid Gaming, Microbial Integration, Bio-digital Interaction

\section{Introduction}

In recent years, hybrid bio-digital games (hereafter called biotic games), which integrate living organisms and biological materials into computer games [1], have been gaining popularity. Amongst over 60 works that are included in van Eck's hybrid biological digital games database [2], which reference works that stretch as far back as the 1940 s, almost half of them have been created only in the last decade. Such games are often driven by the intelligence of non-human organisms (e.g. slime moulds [3, 4]), which offers novel gaming experience in contrast to digital counterparts that are driven solely via computer algorithms. Biotic games also offer not two, but a three-way interaction between humans, computers, and the integrated biological agent, to be explored. However, whilst these features open up a rich design space for interactive entertainment, our current lack of understanding on how players experience games of this nature 
could pose challenges in meaningful improvements of their designs. More specifically, the impact of integrated biological agent in experiencing computer games has not yet been empirically verified and analyzed: Does the integration positively enhance gaming experience over non-integrated equivalent, and how? In this paper, we attempt to answer these questions by presenting and discussing the results of a comparative player study involving two versions of a biotic game called Mould Rush ${ }^{I}$.

Definition. We define the term 'microbial integration' as the physical presence of living micro-organisms in the game's Biotic Processing Unit (BPU) [5]. The BPU of Mould Rush is a modified flatbed scanner described by Kim et al. [6], designed to house the growing microbes and allow continuous imaging of their growth [7].

\section{Related Works}

Microbe-Integrated Systems. In Hossain's user study involving interactive cloud experimentation system and manipulation of slime moulds [8], participants expressed a preference for 'real' experiments involving live microbes, over simulations. Hossain concluded that one of the reasons behind this preference could be due to the implicit narrative attached to real systems, and that it would in turn increase the user's sense of connection to the system. In the user study of Trap It! [9], a touchscreen-based tool that enables playful human-microbe interaction, it was noted that some users expressed excitement upon realizing that they were interacting with real micro-organisms (Euglena $S p$.). The study hypothesized that such realization drove user motivation and interest in further experiencing the system. In Kim's user study of LuduScope [10], an interactive smartphone microscopy for games with Euglena, revealed that when compared to computer-generated simulations, the majority of participants stated benefits of interacting with the real biology ("It is more convincing if you have a real cell"). Although these studies suggest that playing with real organisms would offer better playing experience (e.g. in terms of enjoyment), no comparative study involving real and simulated versions of a biotic game has yet been carried out, in order to verify this hypothesis.

Player Expectations. In van Eck and Lamers' survey on player expectations around biotic games [11], one of their conclusions stated that players expected increased enjoyment when playing against real animals (that are mediated by computers). Further findings also hinted at possible reasons why this would be the case: The majority of the respondents had expected and preferred the added unpredictability offered by the animal opponent, as well as the novelty factor that the game offered. Yet, as acknowledged by van Eck and Lamers ("expectations are not experiences"), a comparative empirical study on actual player experiences is called for, to verify such player expectations.

1 https://biohackanddesign.com/mould_rush/ 
Human vs. Algorithmic Control. In Weibel's study [12], it was found that players had experienced better presence, flow, and enjoyment whilst playing a game with humancontrolled opponent, in comparison to playing with a computer-controlled one. Although the results cannot be directly used to hypothesize that similar results can be replicated with animal/microbe-controlled opponents, this is a relevant study nevertheless, as it measures well-known dimensions of player experience, comparing the effects of biological intelligence (human) and algorithmically-controlled (computer) opponents.

\section{Player Experience Study}

\subsection{Objectives and Hypothesis}

The main objective of the study was to find out how the integration of real microbes affected players' experience of a biotic game. In order to achieve this, two versions of Mould Rush game were tested. First version was used as a control, which did not integrate real mould in the BPU, but had only allowed players to interact with pre-recorded images of its growth. The second version allowed players to interact with real living mould as part of the game. We hypothesized that the players who play with integrated mould would have a more positive overall playing experience than those who did not.

\subsection{Mould Rush Game}

Overview. Mould Rush is a proof-of-concept, online multiplayer game used for the study. The game invites players to watch a live broadcast on w witch $^{2}$, streaming a plate of micro-organisms ('mould') growing in real-time. Graphic overlays are used to divide the plate into numbered segments, which players can select during the game. The goal of the game is to collect as many microbes as possible within an allocated timeframe. Players do this by choosing a segment by typing its number on the game's chat/message box. (fig.1). Players can also reduce the opponents' chances of collecting cells, by either preventing them from collecting cells from a segment (using block command), or by destroying the cells that the opponents had previously collected (using kill command).

Basic Rules \& Scoring. The game lasts for three days. The players are permitted to submit one set of commands (collect $\mid$ block $\mid$ kill) at least once daily, and at most three times daily. Scores from each segment is calculated based on the coverage of microbes found in the segment, calculated using an image recognition script in Open $C V$. At the end of the third day, total number of microbes collected by each player is calculated, with the winner as the player with most accumulation of microbes. Comprehensive background and details of the technical set-up can be found in Mould Rush website ${ }^{1}$.

Versions. Version 1: This consists of pre-recorded timelapse of microbial growth, broken into a series of images that are shown to the player during the game. Players observe

\footnotetext{
${ }^{2}$ https://www.twitch.tv/mould_rush
} 
the growth and simply submit commands to collect, block, and kill cells. All commands are translated into graphical symbols, which are overlaid on top of the growth images (fig.2, left). Note that the growth images are updated every hour, with changes in microbial growth appearing at the same rate as the real growth. The use of pre-recorded images means that exact visual representation of mould can be achieved, and thus they are aesthetically more realistic than graphic illustrations or computer renderings. Version 2: This integrates real microbes into the game's BPU for game play. As such, it live broadcasts real cells growing and dying ("live and alive mode") (fig.1, 2, right).

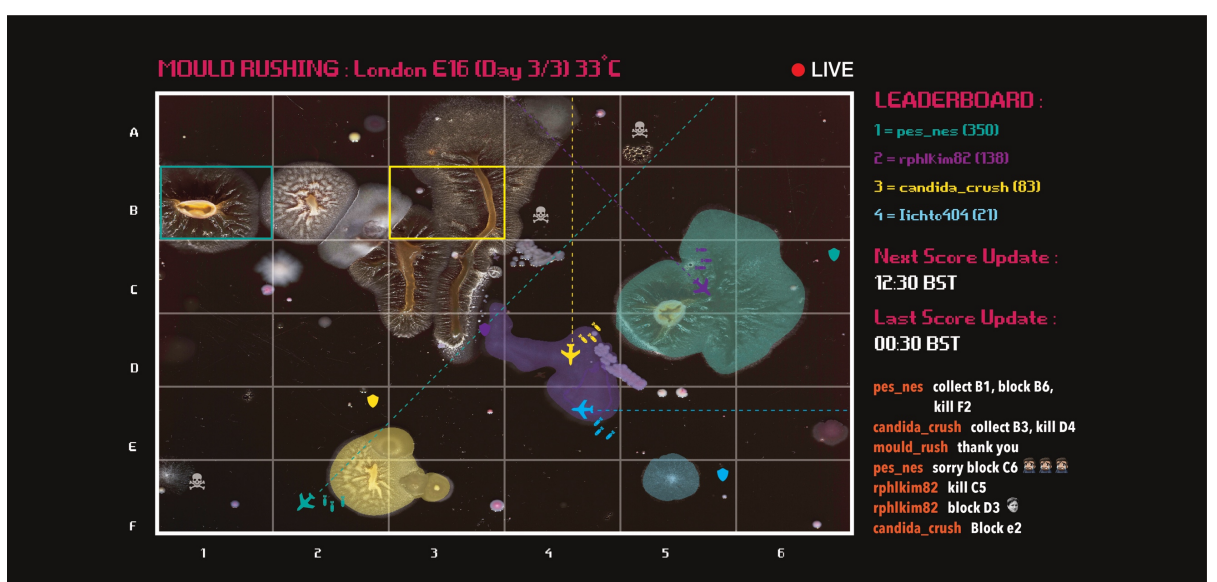

Fig. 1. Scene from a typical Mould Rush game version 2. Note image of microbes growing in real-time, that are divided into identifiable segments. Graphic overlays depict different commands, which are submitted by players through the game chat box (bottom right hand corner).

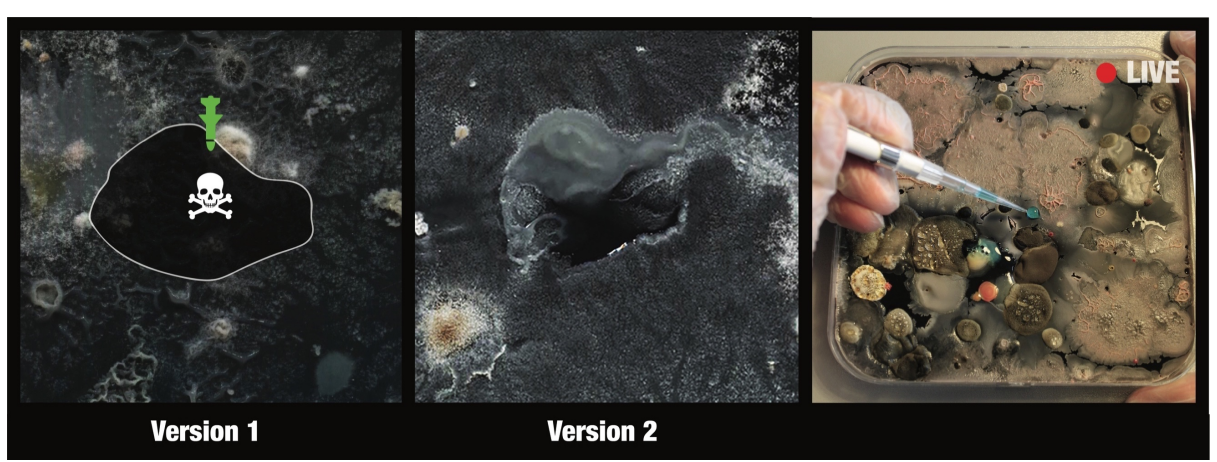

Fig. 2. Kill command in action: Version 1 (left) simulates cell killing with simple graphic overlay, whereas version 2 shows the extent of real physical destruction of cells (right) that had been carried out as part of the live broadcast by the moderator (far right).

The 'Kill' Command. When players decide to kill a segment, microbes found inside the segment are destroyed in one of two ways, depending on the version of the game 
played. In game version 1, the cells are destroyed virtually, only through graphic depiction (fig.2, left). In version 2, the cells are killed in real life, mid-livestream, by the moderator who drops an antibacterial agent (i.e. bleach) onto the segment of the growth plate (fig.2, far right). As a result, those playing with version 2 of the game are able to see the destroyed area in real life (fig.2, right).

\section{$4 \quad$ Materials and Methods}

\subsection{Study Design}

Participants. In total, 40 participants took part in the study (16 female, 26 male, mean age $=25.8, \mathrm{SD}=4.15$ ). They were recruited through adverts placed in the lead author's university email list and on flyers placed around the campus. Participants were a mixture of the University students and staff. All participants received a cash incentive for participating. The participants were divided equally into two cohorts of 20. Cohort 1 played Mould Rush version 1 and cohort 2 played version 2 of the game.

Game Experience Questionnaire (GEQ). Developed by Ijsselsteijn et al. [13], GEQ is a self-reporting questionnaire used to measure various game-related, subjective dimensions of playing experience, which include the following. Core module: Competence; Sensory and Imaginative Immersion; Flow; Tension/Annoyance; Challenge; Negative Affect; Positive Affect. Post-game module: Positive Experience; Negative Experience; Tiredness; Returning to Reality. In order to quantify these dimensions, a set of statements that describe various feelings associated with player experience were presented to the players, for them to relate on a five-point Likert scale. The scale ranged from 0 (Do not agree) to 4 (Completely agree), with example statements including: "I was interested in the game's story"; "I felt frustrated"; and "It was aesthetically pleasing". The Likert ratings of each statement (50 in total) were combined in specific combinations as outlined by Ijsselsteijn et al. [13], and were subsequently averaged to provide the mean GEQ scores for each dimension.

Participant Interview. Each participant was interviewed individually after the game to discuss their experiences. In order to produce a well-rounded picture of their experience, the interviews were intended to complement the GEQ. Each interview lasted around 10 minutes, and were conducted either in person, via phone, Facebook, or Skype. Two open-ended questions were asked to start the interview (Q1: How did you find the game? Q2: Tell me what you liked and disliked about this game, and why?). Participants were asked to elaborate further if they gave single-word or ambiguous statements.

\subsection{Running the Study}

Briefing. Participants were given an information sheet explaining the background of the study, and a consent form to sign. They were also provided with the game rule book. 
Careful measures were taken to ensure that each participant was aware of their game set up, i.e., whether it allowed interaction with live micro-organisms (version 2), or not (version 1). Participants were free to log into the game at any point during the study, using a device of their choice (e.g. desktop/laptop). A day prior to the start of the game, each participant was issued with a Twitch account username and password which enabled them to submit commands on the chat box.

Game Set-Up and Scheduling. A pilot study with four unpaid volunteers from the University was carried out prior to the main study, to ensure consistent game operation. Each game consisted of four competing participants and a moderator (i.e., the lead author). The first day was practice day, to help participants to familiarize with the game and ask any questions to the moderator. The second and third days were proper game days. Each day started at 12:30 BST and ended at 00:30 BST. Score updates were made at the following times: 12:30 BST, 18:30 BST, and 00:30 BST. The moderator was responsible for a) streaming the game on Twitch, b) moderating chat box messages, c) culturing live micro-organisms for version 2 of the game, and d) destroying microorganisms to process the kill commands. The microbes were cultured in a laboratory environment, and participants were not physically exposed to the cells during the study.

\section{$5 \quad$ Results}

\subsection{Mean GEQ Scores}

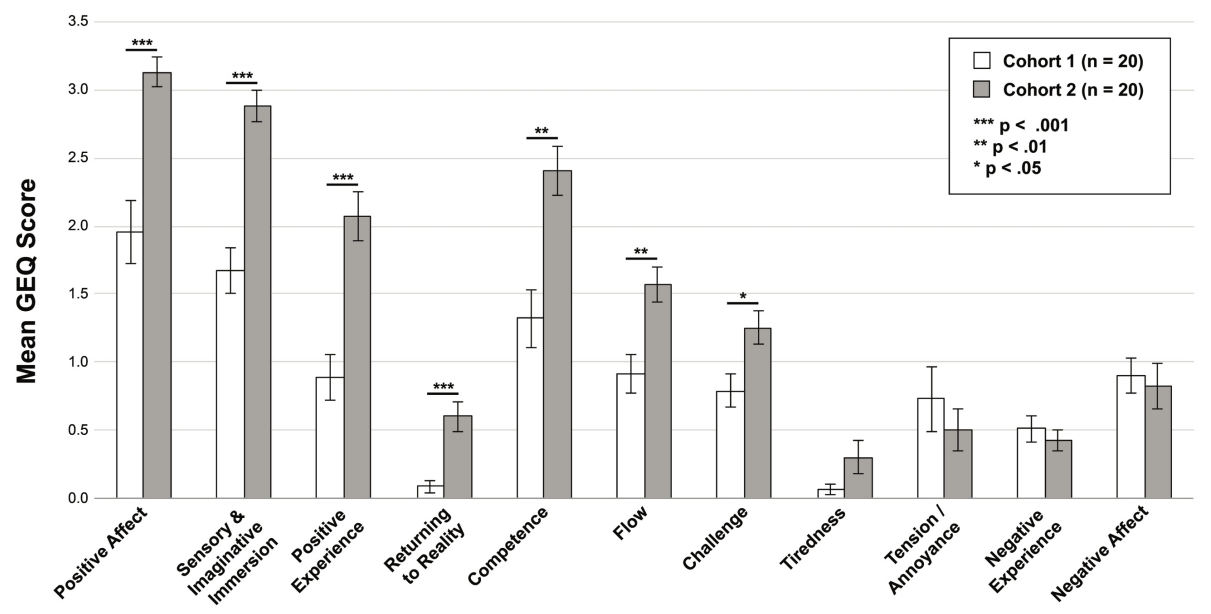

Fig. 3. Mean GEQ scores of 11 player experience dimensions between cohort 1 and 2. Standard error bars are shown. The dimensions are ordered in the order of decreasing significance in terms of their differences in mean GEQ scores between cohorts 1 and 2. 
Positive Affect: Cohort $1(\mathrm{M}=1.96, \mathrm{SD}=1.03)$, Cohort $2(\mathrm{M}=3.13, \mathrm{SD}=0.48) ; \mathrm{t}(38)$ $=4.49, p<.001$. Sensory and Imaginative Immersion: Cohort $1(\mathrm{M}=1.67, \mathrm{SD}=$ $0.76)$, Cohort $2(\mathrm{M}=2.88, \mathrm{SD}=0.51) ; \mathrm{t}(38)=5.66, p<.001$. Positive Experience: Cohort $1(\mathrm{M}=0.89, \mathrm{SD}=0.75)$, Cohort $2(\mathrm{M}=2.07, \mathrm{SD}=0.80) ; \mathrm{t}(38)=4.42, p<.001$. Returning to Reality: Cohort $1(\mathrm{M}=0.09, \mathrm{SD}=0.20)$, Cohort $2(\mathrm{M}=0.60, \mathrm{SD}=$ $0.48) ; \mathrm{t}(38)=3.88, p<.001$. Competence: Cohort $1(\mathrm{M}=1.32, \mathrm{SD}=0.97)$, Cohort 2 $(\mathrm{M}=2.40, \mathrm{SD}=0.81) ; \mathrm{t}(38)=3.59, p=.001$. Flow: Cohort $1(\mathrm{M}=0.92, \mathrm{SD}=0.63)$, Cohort $2(\mathrm{M}=1.57, \mathrm{SD}=0.57) ; \mathrm{t}(38)=3.19, p=.003$. Challenge: Cohort $1(\mathrm{M}=0.79$, $\mathrm{SD}=0.54)$, Cohort $2(\mathrm{M}=1.25, \mathrm{SD}=0.54) ; \mathrm{t}(38)=2.50, p=.018$. Tiredness: Cohort $1(\mathrm{M}=0.07, \mathrm{SD}=0.18)$, Cohort $2(\mathrm{M}=0.30, \mathrm{SD}=0.55) ; \mathrm{t}(38)=1.58, p=.12$. Tension/Annoyance: Cohort $1(\mathrm{M}=0.73, \mathrm{SD}=1.07)$, Cohort $2(\mathrm{M}=0.50, \mathrm{SD}=0.69)$; $\mathrm{t}(38)=-0.78, p=.44$. Negative Experience: Cohort $1(\mathrm{M}=0.51, \mathrm{SD}=0.46)$, Cohort $2(\mathrm{M}=0.43, \mathrm{SD}=0.34) ; \mathrm{t}(38)=-0.57, p=.57$. Negative Affect: Cohort $1(\mathrm{M}=0.90$, $\mathrm{SD}=0.56)$, Cohort $2(\mathrm{M}=0.83, \mathrm{SD}=0.74) ; \mathrm{t}(38)=-0.33, p=.75$.

All participants $(\mathrm{N}=40)$ had completed the GEQ after each game. Mean GEQ scores of 11 player experience dimensions were calculated using the GEQ scoring system [13] and are presented in fig.3. An independent t-test was conducted to compare the mean GEQ scores produced between the two cohorts. Significance threshold was set at 05 . All positive dimensions were shown to be significantly higher in mean GEQ scores in cohort 2 than in cohort 1 (fig.3). This result suggests that when players interact with live organisms during the game of Mould Rush, their experiences are positively enhanced. The complete set of $t$-values and $\mathrm{p}$-values for all dimension are outlined below, with the dimensions ordered in decreasing significance between the two cohorts.

Confirming our hypothesis, the mean GEQ scores show that the players who played Mould Rush with microbial integration had an overall more positive playing experience than those who did not.

\subsection{Participant Interviews}

15 out of 20 participants from cohort 1 took part in the post-game interview $(\mathrm{M}=9.17$ mins) which was conducted over Skype (60\%) and telephone (40\%). All participants from cohort 2 took part in the post-game interview $(\mathrm{M}=10.37 \mathrm{mins})$ which was conducted over Skype (30\%), telephone (20\%) and Facebook messenger (50\%). Participant responses were categorized into themes, and ranked according to their popularity ${ }^{3}$. An emoticon was assigned for each theme according to the nature of the majority of the opinions expressed $(\odot)=$ mostly positive $: *$ = mostly negative $\odot=$ equally positive and negative). An abbreviated summary of the results is shown in tables 1 and 2.

3 Only the top 8 most popular themes have been presented in this paper for brevity. 
Table 1. Abbreviated summary of cohort 1 interviews

\begin{tabular}{|c|c|c|c|}
\hline Rank & Theme & (:) $/:$ & Typical Remark \\
\hline 1 & Game Rules & : & "I didn't like long periods between score updates" \\
\hline 2 & Slowness & : : & "Lack of immediate response from cells was frustrating" \\
\hline 3 & Visuals & ;:) & "The cells looked real and impressive" \\
\hline 4 & Other & $:-$ & "I felt disappointed, no sense of achievement or euphoria" \\
\hline 5 & Tangibility & : : & "I felt very distant from the game" \\
\hline 6 & Unpredictability & ;) & "I liked random shapes that the cells were producing" \\
\hline 7 & Novelty & - & None \\
\hline 8 & Narrative & - & None \\
\hline
\end{tabular}

Table 2. Abbreviated summary of cohort 2 interviews

\begin{tabular}{|c|c|c|c|}
\hline Rank & Theme & :; / : & Typical Remark \\
\hline 1 & Other & ;) & "It was fascinating" \\
\hline 2 & Slowness & (:) & "I enjoyed seeing the mould culture develop over time" \\
\hline 3 & Game Rules & : : & "Space is too limited for full gaming experience" \\
\hline 4 & Visuals & ;) & "The mould produced great colours and shapes" \\
\hline 5 & Novelty & ;) & "A game with an unknown rule set! (microbial growth)" \\
\hline 6 & Unpredictability & ;) & "Relying on randomness of microbes was satisfying" \\
\hline 7 & Tangibility & 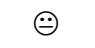 & "I liked the fact that it was against a real organism" \\
\hline 8 & Narrative & (;) & "Watching cells being killed added layer to the story" \\
\hline
\end{tabular}

Common and Contrasting Themes Between the Two Cohorts. As deducted from tables 1 and 2, there were five mutual themes. Among them, Game Rules, Visuals, and Unpredictability had shared the same perception (negative), whereas Slowness and Tangibility were expressed in contrasting manners between the two cohorts.

\section{Discussion}

\subsection{Mean GEQ Scores and Interview Data}

Whilst it is impractical to elaborate on all 11 dimensions that has been measured by GEQ, below we highlight the ones with most compelling implications, for discussion. These are also cross-referenced with the interview data to corroborate our arguments.

Sensory and Imaginative Immersion. As one of the most significantly enhanced GEQ dimension measured in cohort 2 in comparison with cohort 1 , one component statement that forms this dimension include "I was interested in the game's story". This finding supports Hossain's assessment that real systems have implicit narrative attached to them, making it attractive for players to connect with them [8]. In addition to a typical 
interview remark mentioned in table 2, the strong sense of narrative felt by the players are also reflected in comments such as "Showing us the growth plate, and the ritual of killing cells as part of the gameplay, add to the story and makes the game special".

Negative Experiences. Interestingly, none of the negative dimensions from the GEQ (Tiredness; Tension/Annoyance; Negative Experience; Negative Affect) showed significant differences between the two cohorts (fig.3). The interview data points at three common problematic features of Mould Rush game encountered by both cohorts that may explain the phenomena. First one concerns the lack of immediacy in interactivity ("Sometimes the progress could not be seen in real-time and that was frustrating. As gamers, we are used to seeing actions in real-time."). The second feature was the lack of clarity in rules ("I wasn't quite sure how you defined a microbe within a cell to calculate the scores. Because they look so ambiguous, I'm not sure if the scoring system is entirely fair"). And thirdly, the lack of time given for strategizing ("The timescale was too short to formulate a strategy and to gauge other players' styles").

Based on this, we may speculate that the very integration of real microbes in Mould Rush version 2 have not been directly responsible for participants' negative experience.

Further inferences may be made that the addition of real biological element can at best add value to the game, but at worst do not contribute towards negative experience.

However, caution must be exercised when interpreting this observation, as none of the participants had experience of playing biotic games before, and that novelty-bias may have masked out negative aspects of Mould Rush game.

Contrasting Perception of Slowness. The interviews revealed a striking contrast between the two cohorts in how they perceived the slow real-time microbial growth. Whilst the two versions of the game ran at the same speed (i.e. rates of growth were both depicted in real-time), those who played with real microbes felt that the slowness was a contributing factor towards enjoyment ("I looked forward to taking a peep occasionally throughout the day and see small landscape changes happen"), whereas those who did not, had felt that slowness was a hindrance ("I felt frustrated by the lack of dynamic changes on the screen"). Further still, one participant from cohort 1 remarked, "If I could play with real microbes, I would have enjoyed it more as I would have treated it like gardening, or even real hunting (of animals to catch)". We hypothesize that the realization by the players that they are playing with real organisms alters their perception of slowness, perhaps by increasing their tolerance to slow game dynamics, or by associating it to another leisure activity where slowness is expected.

Microbial Aesthetics (Visuals). One of the most consistent and popular remarks made by participants from both cohorts was the visual aspect of Mould Rush ("Growth patterns of mould were colourful and stunning"; "The weird and wonderful shapes they (mould) produced, I felt that I was on an alien planet"). This is a promising observation which offers an empirical evidence to support Gerber's recommendation [5] in designing effective hybrid bio-digital games ("The biological features of interest should be highlighted to the player"). 


\subsection{Bio-Ethics}

During the interview with cohort 2, who had played Mould Rush with real microbes, only one participant $(5 \%)$ had raised concerns about the ethical aspects of the game ("Maybe it would have been better to label 'kill' commands as 'attack' or 'destroy' commands, as I felt uncomfortable being reminded that I was killing living things as part of a game"). Such low number was surprising given that the game involved manipulation (i.e. killing) of living cells for the sake of entertainment. This notion had previously attracted several types of ethical criticisms from the general public, who were reacting to Riedel-Kruse's game involving Paramecia [14]. We hypothesize that the lack of ethical concern displayed with our study may be influenced by the type of microbial species that were being gamified. Whilst Riedel-Kruse's Paramecia are motile and thus display a more animal-like quality, the idea of manipulating mould may less distressing, especially given the negative perception (and apathy) they receive in mainstream media, for instance in adverts for cleaning products. Furthermore, we hypothesize that the remoteness of the microbes' location in relation to the players during gameplay, which had prevented them from physically interacting with microbes, may have reduced potential ethical concerns being raised within the players' minds.

\subsection{Limitations of the Study}

Whilst the implementation of the GEQ had produced a general overview of player experience, the findings may not entirely reflect the experiences that may have been derived exclusively from human-microbe interactions. Since the GEQ had been mainly designed to evaluate conventional computer games (with humans and computers only) [13], experiences associated with non-human biological presence and potential bio-ethical dilemma were not explicitly measured. Therefore, we acknowledge that additional set of questions, included either as an extension to the GEQ and/or the post-game interview, would be beneficial in obtaining a more accurate picture for similar studies in the future. Furthermore, given the high diversity of organisms and interfaces deployed across biotic games [2], we recognize that the findings from our single game-based study may not offer insights that can be translated to all biotic games. As such, we propose that designers approach evaluation of biotic games on a game-by-game, and a species-by-species basis.

\subsection{Wider Implications}

Micro-organisms are common workhorses of synthetic biology [15], and they are likely to increase in significance as the technology advances with time. For example, the increasing sophistication and accessibility of gene editing techniques such as $C R I S P R / \operatorname{Cas} 9$ [16] means that in the future, biotic game designers could build and customize their game characters genetically with an unprecedented granularity. As such, we anticipate a notable increase in the use of microbes in biotic game designs in the next few years, and that this paper can serve as a relevant and insightful case study. 


\subsection{Further Work}

We aim to use our findings as a starting point to further our investigations on specific areas of bio-digital gaming experience. For instance, the contrasting reactions of players from the two different cohorts on slowness of interactivity, will motivate us in testing our hypothesis that the slowness of microbial growth is tolerated (and perhaps even enjoyed) when real organisms are integrated into computer games.

Additionally, Mould Rush is an uncommon type of biotic game, in the sense that it resides within the Internet of Things (IoT) framework, which focusses on the connectedness of an increasing range of physical things via smart devices [17]. Through connecting micro-organisms to an online gaming platform Twitch, Mould Rush provides an opportunity to investigate the effects of remote gameplay and indirect biological manipulation on player experience, as well as on a wider range of socio-cultural benefits that IoT applications can potentially bring $[18,19]$.

\section{Conclusion}

Overall, our study was the first of its kind in scientifically and empirically comparing the feelings and perceptions of players who had engaged in biologically-integrated and non-integrated forms of computer gaming. The findings confirm our initial hypothesis that playing with live interactive function with integration of real micro-organisms enhance the playing experiences of the gamer, in contrast to playing the game's equivalent without integrated micro-organisms. Furthermore, we report on the possible reasons behind such enhancement of player experience. This includes (but not exclusive to): Enhanced narrative through sensory and imaginative immersion, and aesthetic enjoyment through unique visual growth patterns produced by microbial growth. We propose that such observations can be a helpful indicator towards better design of games of this nature, as designers can increase their focus on enhancing their game's narrative, and by highlighting special biological features that are impractical to be emulated or simulated by computers. We also report on significant interview results that can form the basis for further investigation. More specifically, the concept of slowness and how they are perceived between real and simulated gaming is a potentially promising avenue for further investigation. This paper also highlights the need to formulate biology-specific models, to measure player experiences more accurately, which ultimately would help better understanding of bio-digital games in the future and progress the field forward.

Acknowledgements. This research was supported by EPSRC and AHRC Centre for Doctoral Training in Media and Arts Technology (EP/L01632X/1).

\section{References}

1. Riedel-Kruse, I.H., Chung, A.M., Dura, B., Hamilton, A.L., Lee, B.C.: Design, engineering and utility of biotic games. Lab Chip 11(1), 14-22 (2011). https://doi.org/10.1039/ COLC00399A

2. Database: Hybrid Biological Digital Games. https://biodigitalgames.com/database/ 
3. Jabr, F.: How brainless slime molds redefine intelligence. Nature News. 13 November (2012). https://doi:10.1038/nature.2012.11811

4. Slime Mold Andi. https://medium.com/@slime_mold_Andi

5. Gerber, L.C., Kim, H., Riedel-Kruse, I.H.: Interactive biotechnology: design rules for integrating biological matter into digital games. In: DiGRA/FDG (2016).

6. Kim, R., Thomas, S., van Dierendonck, R., Poslad, S.: A new mould rush: designing for a slow bio-digital game driven by living micro-organisms. In: Proceedings of the 13th International Conference on the Foundations of Digital Games (FDG '18) (2018). https://doi.org/10.1145/3235765.3235798

7. van Eck, W., Lamers, M.H.: Biological content generation: evolving game terrains through living organisms. In: Johnson, C., Carballal, A., Correia, J. (eds.) EvoMUSART 2015. LNCS, vol. 9027, pp. 224-235. Springer, Cham (2015). https://doi.org/10.1007/978-3-31916498-4 20

8. Hossain, Z., Jin, X., Bumbacher, E.W., Chung, A.M., Koo, S., Shapiro, J.D., Truong, C.Y., Choi, S., Orloff, N.D., Blikstein, P., Riedel-Kruse, I.H.: Interactive cloud experimentation for biology: an online education case study. In: Proceedings of the 33rd Annual ACM Conference on Human Factors in Computing Systems (2015). https://doi.org/10.1145/2702123.2702354

9. Lee, S.A., Bumbacher, E., Chung, A.M., Cira, N., Walker, B., Park, J.Y., Starr, B., Blikstein, P., Riedel-Kruse, I.H.: Trap it!: a playful human-biology interaction for a museum installation. In: Proceedings of 33rd Annual ACM Conference on Human Factors in Computing Systems (2015). https://doi.org/10.1145/2702123.2702220

10. Kim, H., Gerber, L.C., Chiu, D., Lee, S.A., Cira, N.J., Xia, S.Y., Riedel-Kruse, I.H.: LudusScope: accessible interactive smartphone microscopy for life-science education. PLoS ONE 11(10): e0162602. (2016). https://doi.org/10.1371/journal.pone.0162602

11. van Eck, W., Lamers, M.H.: Player expectations of animal incorporated computer games. In: Chisik Y., Holopainen J., Khaled R., Luis Silva J., Alexandra Silva P. (eds.) Intelligent Technologies for Interactive Entertainment. INTETAIN 2017. Lecture Notes of the Institute for Computer Sciences, Social Informatics and Telecommunications Engineering, vol 215. Springer, Cham (2017).

12. Weibel, D., Wissmath, B., Habegger, S., Steiner, Y., Groner, R.: Playing online games against computer- vs. human-controlled opponents: effects on presence, flow, and enjoyment. Comput. Hum. Behav. 24(5), 2274-2291 (2008). https://doi.org/10.1016/j.chb.2007.11.002

13. IJsselsteijn, W.A., de Kort, Y.A.W., Poels, K.: The Game Experience Questionnaire, Technische Universiteit, Eindhoven (2013).

14. Harvey, H., Havard, M., Magnus, D., Cho, M.K., Riedel-Kruse, I.H.: Innocent fun or 'microslavery'? Hastings Cent. Rep. 44(6), 38-46 (2014). https://doi.org/10.1002/hast.386

15. Zhang, W., Nielson, D.R.: Synthetic biology applications in industrial microbiology. Front. Microbiol.5.(2014). Https://doi.org/10.3389/fmicb.2014.00451.

16. Adli, M.: The CRISPR tool kit for genome editing and beyond. Nature Communications. 9:1911. (2018).

17. Poslad, S.: Ubiquitous Computing: Smart Devices, Environments and Interactions. John Wiley \& Sons Ltd, West Sussex, United Kingdom (2009).

18. Charlton, P., Poslad, S.: A sharable wearable maker community IoT application. In: 12th Int. Conf. on Intelligent Environments, IE'16 (2016).

19. Poslad, S., Ma, A., Wang, Z., Mei, H.: Using a smart city IoT to incentivise and target shifts in mobility behaviour - is it a piece of pie? Sensors 15(6), 13069-13096 (2015). 